NIST Special Publication 1200-23

\title{
Preparation of a Set of Total RNA Benchmarking Samples for Performance Assessment of Genome-scale Differential Gene Expression \\ Version 1.0
}

Lindsay K. Vang

P. Scott Pine

Sarah A. Munro

Marc L. Salit

This publication is available free of charge from: https://doi.org/10.6028/NIST.SP.1200-23 
NIST Special Publication 1200-23

\title{
Preparation of a Set of Total RNA Benchmarking Samples for Performance Assessment of Genome-scale Differential Gene Expression Version 1.0
}

\author{
Lindsay K. Vang \\ P. Scott Pine \\ Sarah A. Munro \\ Marc L. Salit \\ Joint Initiative for Metrology in Biology \\ Material Measurement Laboratory
}

This publication is available free of charge from:

https://doi.org/10.6028/NIST.SP.1200-23

June 2017

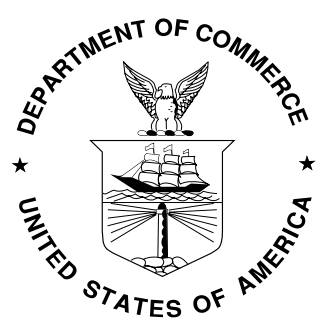

U.S. Department of Commerce Wilbur L. Ross, Jr., Secretary

National Institute of Standards and Technology Kent Rochford, Acting NIST Director and Under Secretary of Commerce for Standards and Technology 
Certain commercial entities, equipment, or materials may be identified in this document in order to describe an experimental procedure or concept adequately. Such identification is not intended to imply recommendation or endorsement by the National Institute of Standards and Technology, nor is it intended to imply that the entities, materials, or equipment are necessarily the best available for the purpose.

National Institute of Standards and Technology Special Publication 1200-23 Natl. Inst. Stand. Technol. Spec. Publ. 1200-23, 11 pages (June 2017)

CODEN: NSPUE2

This publication is available free of charge from: https://doi.org/10.6028/NIST.SP.1200-23 


\section{FOREWORD}

This special publication results from a collaborative research effort between the National Institute of Standards and Technology (NIST) and the Early Detection Research Network $(\mathrm{EDRN})$ of the National Cancer Institute (NCI).

NIST is interested in soliciting feedback on this method. We value user comments and suggestions to improve or further validate this protocol. We also encourage users to report citations of this protocol. Please send your name, email address, and comments to:

Lindsay K. Vang (lindsay.vang@nist.gov)

P.Scott Pine (p.scott.pine@nist.gov)

Sarah A. Munro (sarah.munro@nist.gov)

Marc L. Salit (salit@nist.gov) 


\section{Introduction}

A variety of technologies have been developed for genome-scale profiling of differential gene expression. The level of gene expression difference is typically reported in the form of a ratio, comparing one sample (or class of samples) to another. Studies to develop cancer biomarkers typically involve comparing tumor tissue to normal tissue and identifying features that distinguish the two classes. However, most published cancer biomarkers never make it into clinical practice [1], with a major technical challenge being the irreproducibility of results [2].

A set of papers describes assessing technical performance using sets of mixed total RNA samples designed with between-sample differences in the relative abundance of mRNA [3-7]. Recent work within the Early Detection Research Network (EDRN) of the National Cancer Institute [8] has used these mixed sample sets for measurement assurance of genome-scale microRNA (miRNA) biomarker discovery. This protocol presents a general procedure for the preparation of these mixture sets, for any biomolecule cohort (e.g. mRNA, proteins, etc.), provided mixture components are available that express different abundances of the molecules.

\section{Principles and Scope}

This protocol provides a method for the preparation of two mixtures of total RNA (Mix1 and Mix2) derived from three different pure total RNA sources (RNA1, RNA2, and RNA3) from either commercially available or laboratory prepared total RNA. An overview of the mixing process and the resulting sample sets is shown in Figure 1.

This protocol does not describe a method for the selection of the RNA components. The choice of total RNA to be used for each component should be governed by intended use selection of components with interesting abundance profile differences should be guided by measured or literature results. The protocol and the associated worksheet assume that the user has sufficient material on hand to prepare the specified number of samples.

The proportions of the total RNA components in each mixture are designed such that one RNA component provides a one-to-one comparison, and the remaining components provide two-fold reciprocal fold-changes between mixtures (relative proportions of the components are in the table below).

\begin{tabular}{lcccc} 
& & & \multicolumn{2}{c}{ MIIX1/IVIIX2 } \\
\cline { 2 - 5 } \cline { 4 - 5 } RNA1 & Mix1 & Mix2 & Ratio & Log2 Ratio \\
RNA2 & 0.25 & 0.25 & 1 & 0 \\
RNA3 & 0.25 & 0.50 & 0.5 & -1 \\
\cline { 5 - 6 } & 0.50 & 0.25 & 2 & 1 \\
\hline
\end{tabular}

Table 1. Fractions of each pure total RNA component in each mixture and their corresponding ratios for a two-fold up or down design.

This protocol also does not describe how the samples should be processed or analyzed. Metrics for evaluating similar mixture samples have been described previously [3-7]. 


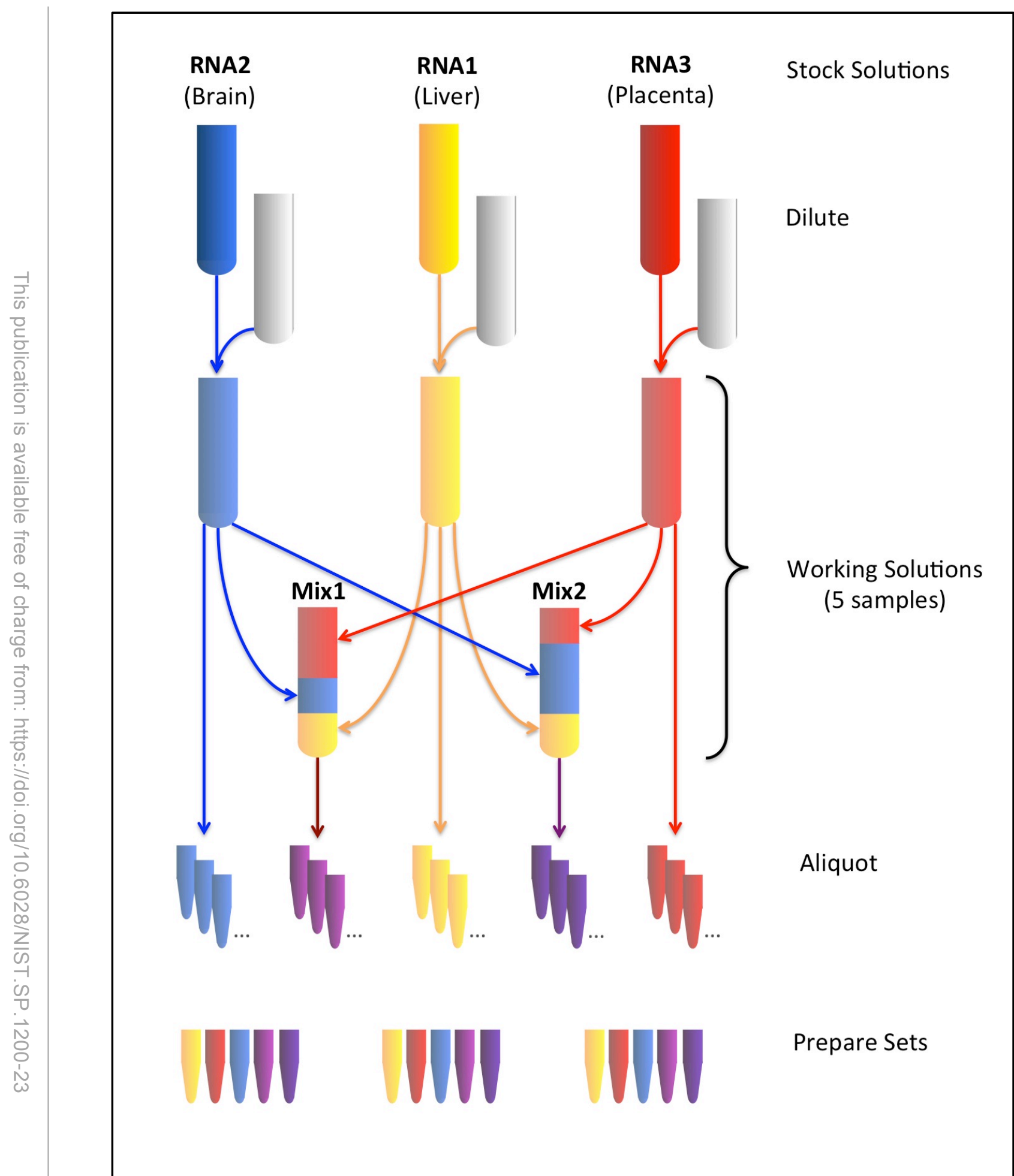

Figure 1. Overview of sample set preparation. Stock solutions of pure total RNA are diluted to provide three working solutions at the concentration required for the final samples. The volume of the pure total RNA working solutions should be sufficient to prepare both mixtures, with sufficient volume remaining for a similar number of pure total RNA aliquots. The attached spreadsheet can be used to determine optimal working solution volumes to provide the required number of sample sets. 


\section{Terminology}

stock solution - concentrated solution of pure total RNA.

working solution - combination of pure total RNA and diluent necessary to produce the final concentration for samples in the sample set.

mixture -pure total RNA working solutions combined in proportions that provide a specific ratio when comparing the two different mixtures.

sample set - a complete set of all samples, including one pure total RNA sample per tissue type and the pair of corresponding mixtures for a total of 5 samples: RNA1, RNA2, RNA3, Mix1 and Mix2.

sample - a single aliquot from the sample set (e.g., one pure total RNA sample or one mixture). 


\section{Reagents, materials, and equipment}

\subsection{Reagents}

4.1.1 RNA1 (e.g., Thermo Fisher Scientific Ambion ${ }^{\text {TM }}$ Human Liver Total RNA)

4.1.2 RNA2 (e.g., Thermo Fisher Scientific Ambion ${ }^{\mathrm{TM}}$ Human Brain Total RNA)

4.1.3 RNA3 (e.g., Thermo Fisher Scientific Ambion ${ }^{\text {TM }}$ Human Placenta Total RNA)

4.1.4 Diluent (e.g., Thermo Fisher Scientific Ambion ${ }^{\mathrm{TM}}$ THE RNA Storage Solution, $1 \mathrm{mM}$ sodium citrate, $\mathrm{pH} 6.4$ )

\subsection{Materials}

4.2.1 RNase-free microcentrifuge tubes with O-ring screwcap (e.g., VWR Polypropylene microcentrifuge tube with polypropylene O-ring screwcap, $0.5 \mathrm{~mL}$, sterile)

Note - Microcentrifuge tubes for volumes $\leq 0.5 \mathrm{~mL}$ should be used due to the small volume of sample being stored. Tubes with larger volume capacity increase the risk of sample loss to the tube walls and evaporation during freezing. It is important for sample integrity that tubes are RNase free and sterile. O-ring caps are recommended to prevent evaporation during storage.

4.2.2 Cryolabels suitable for storage at $-80^{\circ} \mathrm{C}$ (e.g., Diversified Biotech $33 \mathrm{~mm}$ x $13 \mathrm{~mm}$ Cryo Babies labels and $9.5 \mathrm{~mm}$ Tough Spots)

4.2.3 RNase decontamination solution (e.g., Thermo Fisher Scientific RNase-Zap AM9780)

4.2.4 $1.5 \mathrm{~mL}$ and $2.0 \mathrm{~mL}$ Nuclease-free microcentrifuge tubes

4.2.5 Boxes/bags for storage and distribution of sample sets

\subsection{Equipment}

4.3.1 Calibrated pipettes and disposable filtered pipette tips

Note - Single channel pipettes with the following volume range are recommended: $0.1 \mu L-2$ $\mu L ; 2 \mu L-20 \mu L ; 20 \mu L-200 \mu L ; 100 \mu L-1000 \mu L$

Note - Filtered pipette tips are used to avoid the introduction of RNases and crosscontamination of samples.

4.3.2 Vortex mixer

4.3.3 Microcentrifuge

4.3.4 Spectrophotometer or fluorospectrometer capable of measuring RNA concentration (e.g., Thermo Fisher Scientific Qubit ${ }^{\mathrm{TM}}$ or equivalent)

4.3.5 Agilent Bioanalyzer or equivalent Note - The Bioanalyzer is used to measure RNA integrity prior to the pooling of samples. The RIN is a metric calculated by the Agilent Bioanalyzer [9], but another instrument capable of measuring RNA integrity in a similar manner can be used as well. 


\section{Preparation of total RNA benchmarking sample sets}

\subsection{Prepare the worksheet for working solutions and mixtures}

Note-For automatic calculation of values to be entered below, download the spreadsheet associated with this protocol at https://doi.org/10.6028/nist.sp.1200-23s.

5.1.1 Determine the desired sample mass, volume, and total number of sample sets, and input them in the included NIST_SP_Worksheet.

5.1.2 Verify there is sufficient RNA to prepare the specified number of sample sets.

\subsection{Verify concentration and integrity of stock solutions}

5.2.1 Prepare the workspace for work with RNA by wiping down all laboratory benches, pipettes, and gloves with an RNase decontamination solution, according to the manufacturer's specifications.

5.2.2 Thaw the stock solution tubes on ice. Once thawed, briefly centrifuge to collect all of the liquid in the bottom of the tubes.

Note - All tubes containing RNA should be kept on ice while being handled in the lab.

5.2.3 Label an RNase-free $1.5 \mathrm{~mL}$ microcentrifuge tube for each tissue type.

5.2.4 Prepare a $100 \mathrm{ng} / \mu \mathrm{L}$ and a $50 \mathrm{ng} / \mu \mathrm{L}$ dilution of each RNA tissue type. Mix the dilutions thoroughly by pipetting up and down 10 times, then centrifuge briefly to collect the liquid in the bottom of the tube.

5.2.5 Measure $2 \mu \mathrm{L}$ of each RNA dilution using the Qubit ${ }^{\mathrm{TM}}$ or equivalent, according to the manufacturer's specifications. Enter the concentration of the stock solutions in the NIST_SP_worksheet.

5.2.6 Determine RNA quality by running $1 \mu \mathrm{L}$ of the $50 \mathrm{ng} / \mu \mathrm{L}$ RNA dilution on an RNA 6000 Nano Bioanalyzer chip, following manufacturer's specifications.

Note - A Certificate of Analysis (COA) may be available from the total RNA solution provider. The COA may include a Bioanalyzer trace and/or rRNA ratio. Discard the stock solution if the Bioanalyzer trace shows signs of degradation, or the rRNA ratio is significantly different than what is provided in the COA.

\subsection{Prepare the working solutions}

5.3.1 To prepare a working solutions for each component RNA, combine the following in a labeled $2 \mathrm{~mL}$ RNase-free microcentrifuge tube on ice:

Note - Fill the blanks from the lines referenced in the NIST_SP_Worksheet

$\mu \mathrm{L}$ stock solution of RNA1 into

$\mu \mathrm{L}$ diluent for RNA1

$\mu \mathrm{L}$ stock solution of RNA2 into $\mu \mathrm{L}$ diluent for RNA2

$\mu \mathrm{L}$ stock solution of RNA3 into $\mu \mathrm{L}$ diluent for RNA3

5.3.2 On ice, add diluent to each microcentrifuge tube.

5.3.3 Add the full volume of each stock solution to the diluent.

5.3.4 Gently mix the working solutions by vortexing, then centrifuge briefly and place on ice. 


\subsection{Prepare the mixtures}

5.4.1 To prepare a working solution for Mix 1 combine the following in a $1.5 \mathrm{~mL}$ RNasefree microcentrifuge tube on ice:

Note - Fill the blanks from the lines referenced in the NIST_SP_Worksheet

$\mu \mathrm{L}$ RNA1 working solution

$\mu \mathrm{L}$ RNA2 working solution

$\mu \mathrm{L}$ RNA3 working solution

5.4.2 Gently mix the working solutions by vortexing, then centrifuge briefly and place on ice.

5.4.3 To prepare a working solution for Mix 2 combine the following in a $1.5 \mathrm{~mL}$ RNasefree microcentrifuge tube on ice:

Note - Fill the blanks from the lines referenced in the NIST_SP_Worksheet

$\mu \mathrm{L}$ RNA1 working solution

$\mu \mathrm{L}$ RNA2 working solution

$\mu \mathrm{L}$ RNA3 working solution

5.4.4 Gently mix the working solutions by vortexing, then centrifuge briefly and place on ice.

\subsection{Aliquot and package the sample sets}

5.5.1 Prior to preparing aliquots, pre-label all $0.5 \mu \mathrm{L}$ microcentrifuge tubes and O-ring caps necessary for the desired number of sample sets.

5.5.2 Using a calibrated pipette with a volume range $\leq 20 \mu \mathrm{L}$ and filtered pipette tips dispense aliquots of the working solutions into pre-labeled tubes and cap. Complete all aliquots for a given sample before proceeding to the next.

Note-All working solution and pre-labeled sample tubes should be kept on ice for this step. Note - Ensure caps are securely tightened to prevent evaporation during storage.

5.5.3 Sort labeled tubes into sample sets and package for storage.

5.5.4 Store complete sample sets at $-80{ }^{\circ} \mathrm{C}$. 


\section{Abbreviations}

NIST National Institute of Standards and Technology

EDRN Early Detection Research Network

NCI National Cancer Institute

RNA ribonucleic acid

mRNA messenger RNA

miRNA microRNA 


\section{References}

1. Kern SE. Why your new cancer biomarker may never work: recurrent patterns and remarkable diversity in biomarker failures. Cancer Res. 72(23):6097-101 (2012).

2. Plant $\mathrm{AL}$, et al. Improved reproducibility by assuring confidence in measurements in biomedical research. Nat Methods. 11(9):895-8 (2014).

3. Thompson KL, et al. Use of a mixed tissue RNA design for performance assessments on multiple microarray formats. Nucleic Acids Res. 33(22):e187 (2005).

4. Pine PS, et al. Use of diagnostic accuracy as a metric for evaluating laboratory proficiency with microarray assays using mixed-tissue RNA reference samples. Pharmacogenomics. 9(11):1753-63 (2008).

5. Thompson KL and Pine PS. Comparison of the diagnostic performance of human whole genome microarrays using mixed-tissue RNA reference samples. Toxicol Lett. 186(1):58-61 (2009).

6. Pine PS, Rosenzweig BA, Thompson KL. An adaptable method using human mixed tissue ratiometric controls for benchmarking performance on gene expression microarrays in clinical laboratories. BMC Biotechnol. 11:38 (2011).

7. Parsons $\mathrm{J}$, et al. Using mixtures of biological samples as process controls for RNAsequencing experiments. BMC Genomics. 16:708 (2015).

8. Srivastava S and Kramer BS. Early detection cancer research network. Lab Invest. 80(8):1147-8 (2000).

9. Agilent Publication 5989-1165EN. RNA Integrity Number (RIN) - Standardization of RNA Quality Control. 